\title{
An optimized compensation strategy of DVR for micro-grid voltage sag
}

\author{
Zhengming Li*, Wenwen Li and Tianhong Pan
}

\begin{abstract}
Introduction: This paper uses a dynamic voltage restorer (DVR) to improve the voltage quality from voltage sags. It is difficult to satisfy various of compensation quality and time of the voltage sag by using single compensation method. Furthermore, high-power consumption of the phase jump compensation increases the size and cost of a dynamic voltage restorer (DVR).

Methods \& Results: In order to improve the compensating efficiency of DVR, an optimized compensation strategy is proposed for voltage sag of micro-grid caused by interconnection and sensitive loads. The proposed compensation strategy increases the supporting time for long voltage sags.

Discussion: Firstly, the power flow and the maximum compensation time of DVR are analyzed using three basic compensation strategies. Then, the phase jump is corrected by pre-sag compensation. And a quadratic transition curve, which involves the injected voltage phases of pre-sag strategy and minimum energy strategy, is used to transform pre-sag compensation to minimum energy compensation of DVR.

Conclusions: The transition utilizes the storage system to reduce the rate of discharge. As a result, the proposed strategy increases the supporting time for long voltage sags. The analytical study shows that the presented method significantly increases compensation time of DVR. The simulation results performed by MATLAB/SIMULINK also confirm the effectiveness of the proposed method.
\end{abstract}

Keywords: Micro-grid, Voltage sag, Dynamic voltage restorer, Compensation time, Optimized compensation strategy

\section{Introduction}

Since micro grid can integrate various kinds of renewable energy resources, it has been widely investigated in recent years [1]. However, there also exists some power quality problems that cannot be ignored, such as voltage sag, swell, and harmonic [2]. Furthermore, micro grid contains a variety of powers, sensitive loads and non-linear power electronic devices. As a result, the frequent start-up and shutdown operation of renewable power generators will lead to voltage fluctuations, especially sag. The voltage sag can damage the power quality, may even cause huge economic losses [3]. To improve and manage the power quality of micro grid, the flexible alternating current transmission technology (FACT) has been introduced, such as SVC, D - STATCOM, DVR and UPQC [4-6].

\footnotetext{
* Correspondence: Izming@ujs.edu.cn

School of Electrical and Information Engineering, Jiangsu University, Zhenjiang 212000, Jiangsu Province, China
}

Among these devices, DVR is one of the most powerful apparatus that can protect voltage-sensitive equipment from voltage sag, swell and flicker. In some cases, it can also suppress the voltage harmonics and unbalanced three-phase voltage effectively [7].

DVR needs some power exchange when it is used in compensation. In order to reduce power exchange during the effective compensation of voltage drop, the compensation strategy of DVR should be optimized [8]. The present research and application of DVR compensation strategies for voltage sag are pre-sag compensation, in-phase compensation and the minimum energy compensation [9-12]. In-phase compensation method can compensate the sagged voltage with minimum amplitude [9]. The minimum energy method adjusts the injected active and reactive power of DVR to reduce consumption of the dc stored energy [13]. However, both can't correct the phase jump, even lead to trip of sensitive loads. The pre-sag compensation can 
fully restore the amplitude and phase angle of the sagged load voltage [14]. Though pre-sag compensation can guarantee the continuity of grid voltage, the compensation of phase jump needs a large amount of active power in an energy storage system.

Taking advantage of three basic compensation strategies, some comprehensive compensation strategies $[15,16]$ were proposed. The comprehensive strategy of pre-sag and inphase compensation [15] reduced the injected voltage while extending compensation time, but it sustainably used the energy of dc unit. The control that combined pre-sag method and minimal energy method [16] were lacked of the time control though it made good use of dc energy. To avoid these drawbacks, an optimized strategy based on the strategy of paper [16] is discussed in this paper. Firstly, the DVR compensating voltage and power flow for three basic strategies is analyzed and the maximum compensation time is derived from the analysis. Then, pre-sag strategy is used to correct phase jump at the beginning of compensating. According to a quadratic transition curve, the injected phase angle varied from pre-sag to the minimum energy compensation is rotated in multi-step. The rest process is completed by the minimum energy strategy. Owing to the introduction of the transition process, the compensation time of the comprehensive strategy is significantly increased. The performance of the proposed method is validated by using MATLAB/Simulink simulation.

\section{Discussion}

\section{Three basic compensation strategies}

As shown in Fig. 1, DVR system consists of the capacitor (storage device), inverter circuit, LC filter circuit, series transformer. When the voltage sag happens in grid, the dc voltage in energy storage system is converted to ac voltage by the inverter. Then, the ac voltage with a certain amplitude and phase is injected into grid feeder through filter and transformer to restore the sagged load voltage. These operations ensure normal operation of sensitive loads.
As shown in Fig. 2, phase diagrams are applied to analyze characteristics of three basic compensation methods. The Fig. 2(a) shows the in-phase compensation, the Fig. 2(b) and 2(c) shows the minimum energy compensation. The Fig. 2(d) shows the pre-sag compensation. The amplitude and phase angle of DVR compensating voltage are given in the diagrams respectively. In Fig. 2, $U_{G}$ and $U_{S}$ are the system voltages before and after the sag, $U_{L}$ and $U_{A}$ are the load voltages before and after the sag, $U_{D V R}$ is the DVR output compensating voltage, $P_{D V R}$ and $Q_{D V R}$ are the DVR active and reactive power injected to system, $\delta$ is the phase jump of sagged voltage, $\theta_{L}$ is the angle of load power factor. $I_{L}$ is the load current which is set as the reference vector. $\Delta U$ is set as voltage sag depth, so $\Delta U=\left(U_{L}-U_{S}\right) / U_{L}$.

\section{In-phase compensation}

For in-phase compensation strategy, the DVR compensating voltage has the same phase with the sagged grid voltage. The amplitude of compensating voltage is equal to the difference between the reference voltage of load and the grid voltage (shown in Fig. 2(a)). This compensation strategy only needs to measure instantaneous voltage in the grid, so it has high speed. Though DVR injects the minimum compensating voltage amplitude, it cannot correct phase jump of voltage sag. In this case, it may lead to interruption of load voltage. The DVR compensating voltage amplitude and phase angle injected to the power system are:

$$
\begin{aligned}
& U_{D V R}=\sqrt{\frac{2}{3}} U_{L} \Delta U \\
& \angle U_{D V R}=\theta_{L}
\end{aligned}
$$

\section{Minimum energy compensation}

In reactive power compensation, the injected voltage of DVR is orthogonal to load current. This strategy only provides reactive power to compensate voltage sag without

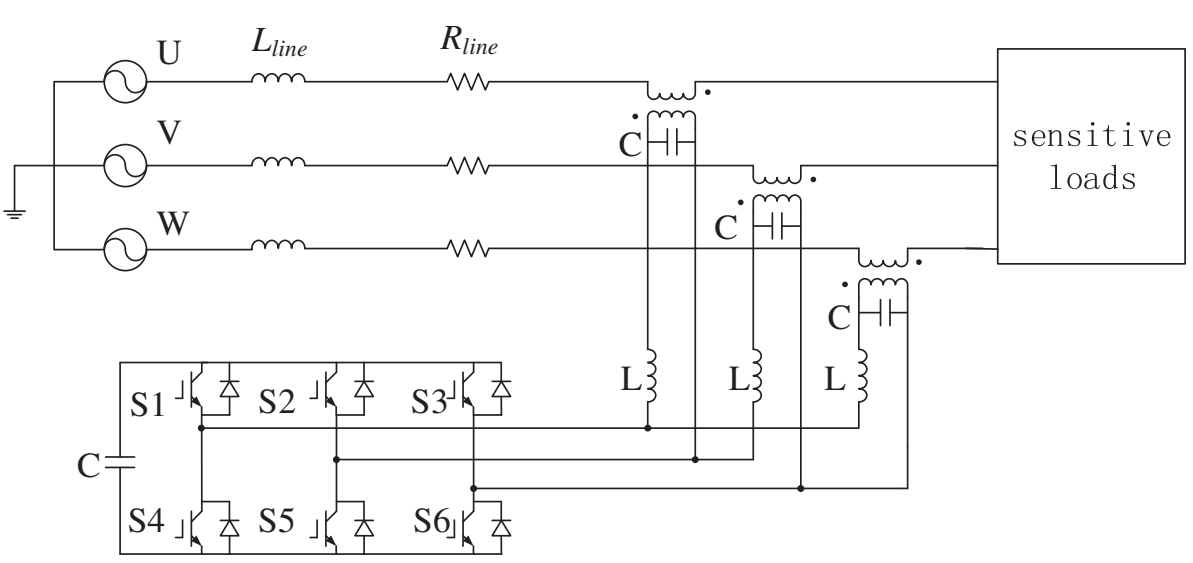

Fig. 1 Topology of DVR 


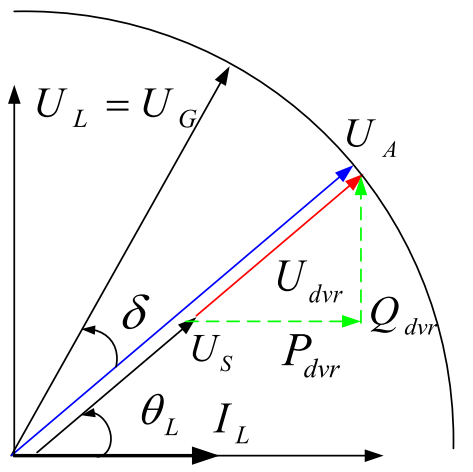

(a)

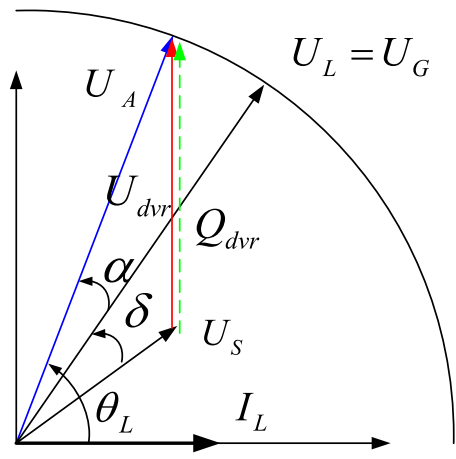

(b)

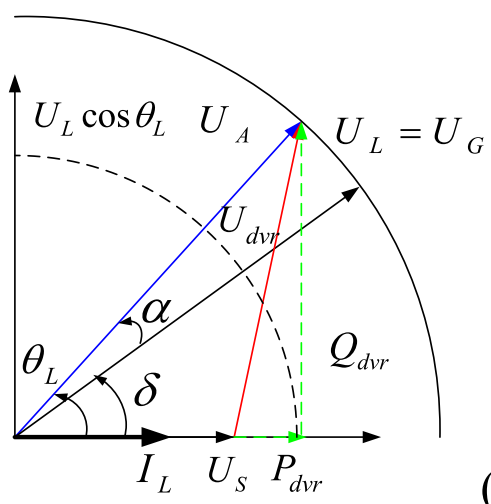

(c)

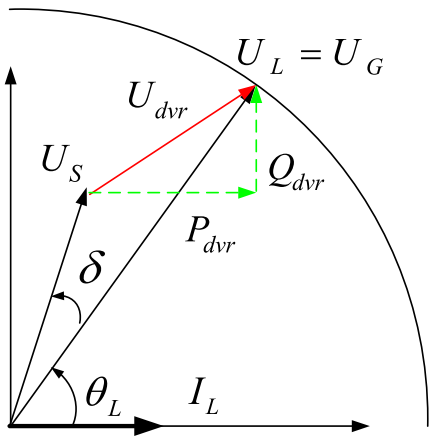

(d)

Fig. 2 Phasor diagram for three basic compensation Strategies active power consumption. As shown in Fig. 2(b), the DVR injected voltage amplitude and phase in the system are:

$$
\begin{aligned}
& U_{D V R}=\sqrt{\frac{2}{3}} U_{L} \sqrt{1-2(1-\Delta U) \cos (\alpha+\delta)+(1-\Delta U)^{2}} \\
& \angle U_{D V R}=\frac{\pi}{2}
\end{aligned}
$$

where $\alpha$ is the phase change caused by DVR reactive compensation. The maximum sag depth in reactive power compensation, $\Delta U_{\max }$, is closely related to the load power factor:

$$
\Delta U_{\max } \leq\left(1-\cos \theta_{L}\right)
$$

When the sagged voltage of grid $U_{S}$ is in phase with load current $I_{L}$, the compensating voltage reaches the maximum value, i.e:

$$
U_{\max }=\frac{U_{S}}{1-\Delta U_{\max }} \sin \theta_{L}
$$

When the voltage sag depth is over the boundary of Eq. (5), DVR must inject some active power into the system of grid to maintain compensation. In this situation, the minimum energy method is put forward to enhance the performance of reactive power compensation. As shown in Fig. 2(c), the amplitude and phase of DVR injected voltage are:

$$
\begin{aligned}
& U_{D V R}=\sqrt{\frac{2}{3} U_{L}} \sqrt{1-2(1-\Delta U) \cos \theta_{L}+(1-\Delta U)^{2}} \\
& \angle U_{D V R}=\tan ^{-1}\left(\frac{U_{L} \sin \theta_{L}}{U_{L} \cos \theta_{L}-U_{S}}\right)
\end{aligned}
$$

\section{Pre-sag compensation}

When the voltage sag happens in the grid system, the phase jump often accompanies with the decrease of voltage amplitude. These methods mentioned above can't compensate the phase jump. Pre-sag compensation ensures that the amplitude and phase of compensating voltage are completely in accord with the pre-sag voltage. However, the storage device has to provide abundant energy in pre-sag compensation. As shown in Fig. 2(d), the amplitude and phase of DVR injected voltage are:

$$
\begin{aligned}
& U_{D V R}=\sqrt{\frac{2}{3}} U_{L} \sqrt{1-2(1-\Delta U) \cos \delta+(1-\Delta U)^{2}} \\
& \angle U_{D V R}=\tan ^{-1}\left(\frac{U_{L} \sin \theta_{L}-U_{L} \sin \left(\theta_{L}+\delta\right)}{U_{L} \cos \theta_{L}-U_{S} \cos \left(\theta_{L}+\delta\right)}\right)
\end{aligned}
$$

\section{Control requirements}

The DVR active power injected to the system is equal to the difference between the active power in the grid and 
load. From Fig. 2(a), (c) and (d), the active power of presag compensation $\left(P_{\text {pre }}\right)$, in-phase compensation $\left(P_{\text {in-phase }}\right)$ and the minimum energy compensation $\left(P_{\text {opt }}\right)$ can be expressed as:

$$
\begin{aligned}
& P_{\text {pre }}=\sqrt{3 U_{L} I_{L}}\left(\cos \theta_{L}-(1-\Delta U) \cos \left(\theta_{L}-\delta\right)\right) \\
& P_{\text {in-phase }}=\sqrt{3 U_{L} I_{L} \Delta U \cos \theta_{L}} \\
& P_{\text {opt }}=\sqrt{3 U_{L} I_{L}\left(\cos \theta_{L}-1+\Delta U\right)}
\end{aligned}
$$

The relation between the DVR active power and other variables (sag depth, phase jump, the load power factor) can be obtained from the three equations. It can be seen that the pre-sag strategy has the highest power consumption among three compensation methods. The operations of the pre-sag compensation require a plenty of energy in dc energy-storage capacitor. In practice, the energy storage of DVR is limited. When the output power reaches a threshold, the compensating voltage will drop. In order to ensure appropriate operations of DVR, it should be satisfied:

$$
\frac{U_{D V R}}{n_{t}} \leq \frac{m_{i \max } U_{d c}}{2}
$$

where $n_{t}$ is the turns ratio of series transformer, $m_{\text {imax }}$ is the maximum modulation index of the DVR inverter, $U_{d c}$ is the DVR dc voltage. Once the dc voltage decreases to the minimum threshold value, that is, over the boundary in Eq. (14), DVR will stop compensating process to avoid harmonic pollution of load voltage. The initial energy stored in dc capacitor is:

$$
W_{d c}=\frac{1}{2} C_{d c} U_{d c}^{2}
$$

After a period of time $\Delta t$, the value of voltage in dc container decreases from $U_{d c}$ to $U_{d c}$. The variation of voltage is $\Delta U_{d c}$. The final energy stored in the capacitor at this time is:

$$
W_{d c f}=\frac{1}{2} C_{d c} U_{d c f}^{2}
$$

During $\Delta t$, the total active power provided by capacitor in steady state is:

$$
\begin{aligned}
P_{d c} & =\frac{W_{d c}-W_{d c f}}{\Delta t}=\frac{1}{2} C_{d c} \frac{d}{d t} U_{d c}^{2} \\
& =\frac{1}{2} C_{d c}\left(\frac{U_{d c}^{2}-U_{d c f}^{2}}{\Delta t}\right)
\end{aligned}
$$

In an ideal system, the DVR dc power in Eq. (11) is equal to the ac power in Eq. (17), so the capacity of capacitor $\mathrm{C}$ can be obtained from the relation. However, the dc voltage will decrease with the flow of power, so as the output compensating voltage. Accordingly, the DVR output active power is also limited by the capacity of dc capacitor and the minimal dc voltage, which is sufficient for a proper restoration of the load voltage. In addition, the dc voltage gradient $d U_{d c} / d t$ is proportional to the DVR injected active power $P_{D V R}$ directly. The less $P_{D V R}$ is, the smaller the slope of dc voltage is. Hence, the compensating time is extended. It can be known that improving the rate of dc voltage drop can prolong the compensation time.

According to Eq. (14) and Eq. (17), the maximum compensation time can be expressed as:

$$
t_{\max }=\frac{C *\left[U_{d c}^{2}-\left(\frac{2 * U_{D V R}}{m_{i \max } * n_{t}}\right)^{2}\right]}{2 * P_{D V R}}
$$

The maximum compensation time $t_{\max }$ directly reflects the utilization level of stored energy in dc capacitor.

\section{Methods \\ The optimized compensation strategy}

The dc voltage gradient is controlled by adjusting the magnitude of DVR injected active power to extend the DVR compensating time. Firstly, pre-sag compensation is used to restore the amplitude and phase angle of sagged voltage. Then a transition process is introduced to shift pre-sag strategy to the minimum energy strategy gradually. The transition avoids direct jump of phase angle at running point. This process is usually implemented in one to two cycles, and the sagged voltage can be restored to stabilization in a short time. The compensating control process is described in detail as follows.

\section{The pre-sag compensation}

In order to reduce the voltage distortions at the load side, the sagged voltage should be restored to the normal value by the pre-sag compensation. After a certain time, the modulation index will increase as a result of discharging of the dc capacitor. If there is no sufficient energy to support the compensation, DVR will not be able to compensate properly. Therefore, as soon as a certain modulation index is reached, a smooth transition from pre-sag compensation to the minimum energy compensation is initiated to reduce consumption of energy.

To detect phase jump, two phase lock loops (PLLs) are adopted at the load side and the grid side respectively. Once the voltage sag is detected, the initial injected angle of DVR should be determined to compensate the load phase jump. On one hand, the pre-sag voltage angle is locked by freezing the PLL at the load side. On the other hand, the phase angle of the sagged grid voltage is locked through the PLL at the grid side. The two angles are equal when the system is steady. When 
the sag happens, the difference between the two phases is equal to the phase angle jump of sagged voltage. Hence, the initial phase angle of DVR injected into the system is:

$$
\theta_{\text {first }}=\tan ^{-1}\left(\frac{U_{L} \sin \theta_{L}-U_{s} \sin \left(\theta_{L}+\delta\right)}{U_{L} \cos \theta_{L}-U_{s} \cos \left(\theta_{L}+\delta\right)}\right)
$$

The voltage sag can be detected according to space vector method. Therefore, the sag depth is regarded as the absolute difference between the load reference voltage (1p.u.) and the actual grid voltage (p.u.). Under the synchronous reference frame, the sag depth can be expressed as:

$$
\Delta U=\left|1-\sqrt{U_{G d}^{2}+U_{G q}^{2}}\right|
$$

\section{The transition process}

After the sagged voltage recovers to normal, pre-sag compensation will be smoothly transformed to the minimum energy compensation in one to two cycles.

From section 1.2, it can be known that the minimum energy method works in two situations. For shallow sag, DVR will make reactive power compensation, in which DVR works in self-supporting mode. It hardly absorbs active power from the grid, or only consumes a little active power to overcome losses in system and maintain the constant voltage in dc capacitor. For deep sag, DVR must carry on the minimum energy compensation. DVR will inject a certain amount of active power to grid, so the energy and voltage in the capacitor will be reduced. In order to maintain the needed output voltage of the inverter, the modulation index will increase until it reaches the maximum value in Eq. (14). Hence, the final injected angle of DVR $\theta_{\text {final }}$ is:

$$
\theta_{\text {final }}= \begin{cases}\frac{\pi}{2}+\varepsilon, & \Delta U \leq\left(1-\cos \theta_{L}\right) \\ \pi-\tan ^{-1}\left(\frac{U_{L} \sin \theta_{L}}{U_{L} \cos \theta_{L}-U_{S}}\right), & \Delta U>\left(1-\cos \theta_{L}\right)\end{cases}
$$

where $\varepsilon$ is the angle change caused by component loss, which can be obtained by PI controller.

The smooth transition of the load voltage will be done by a quadratic function curve to reduce the influence of phase jump to sensitive loads. The curve between the initial and terminal phase is given as:

$$
\theta_{\text {tran }}=\frac{\theta_{\text {first }}-\theta_{\text {final }}}{\Delta t_{\text {tran }}^{2}}\left(t-\Delta t_{\text {tran }}\right)^{2}+\theta_{\text {final }}
$$

where $\Delta t_{\text {tran }}$ is the transitional time.

\section{The operation process}

In Fig. 3, Phase diagrams of the compound control strategy are given. Figure 3(a) shows the voltage amplitude and phase of the pre-sag strategy, and 3(c) shows the voltage amplitude and phase of the minimum energy strategy, (3b) shows the transition process. As shown in Fig. 3, the sag depth is over the limit in Eq. (5), and it produces a positive phase jump with voltage sag. Here, pre-sag strategy is used to recover the amplitude and phase of the sagged voltage. Once the dc voltage or the modulation index reaches the limited value, a transition process is introduced to avoid direct phase jump at the operating point. The compensating voltage will be gradually transformed to the value of the minimum energy compensation. In the transition process, the reactive power of DVR increases gradually, so as the amplitude and phase angle

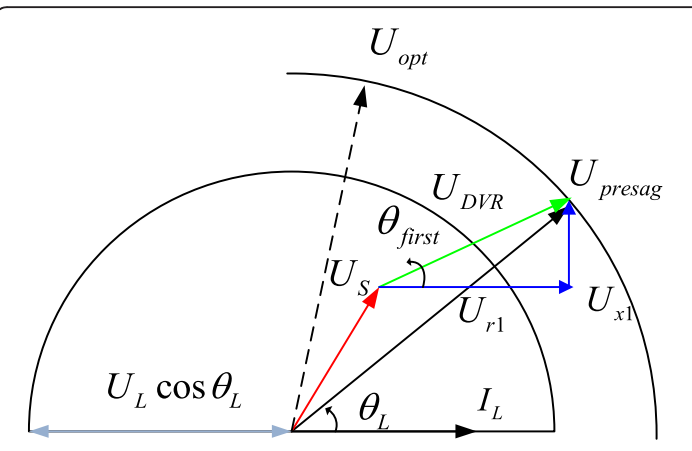

(a)

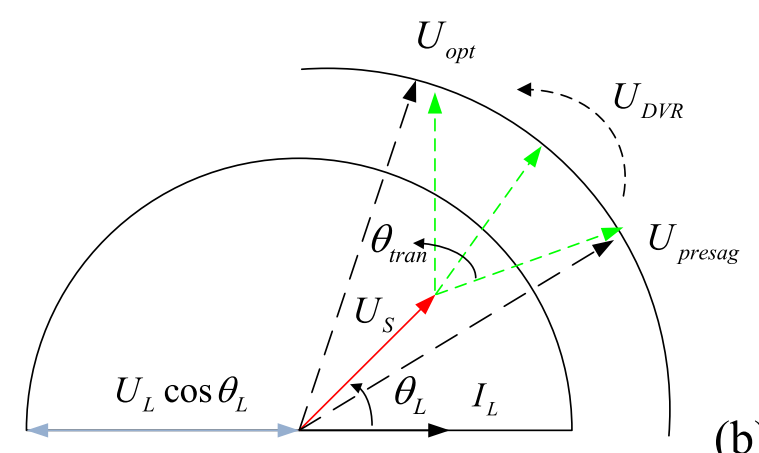

(b)

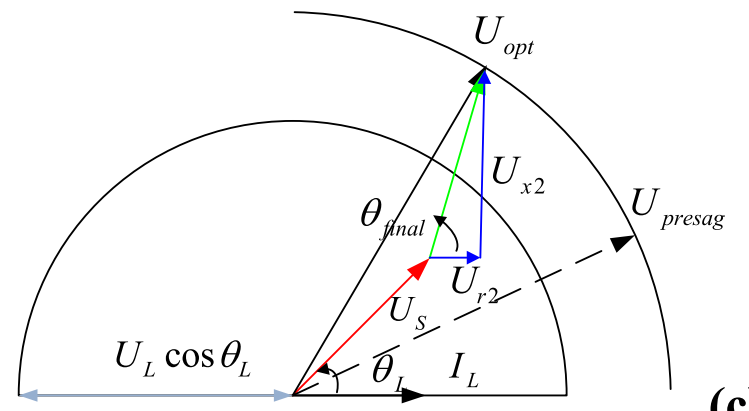

(c)

Fig. 3 Phase diagrams for the compound control strategy 


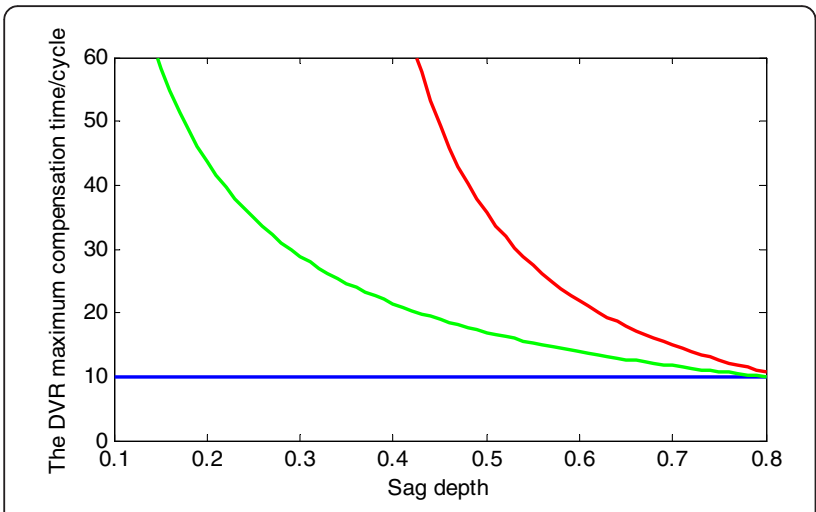

(a) The maximum compensation time and sag depth

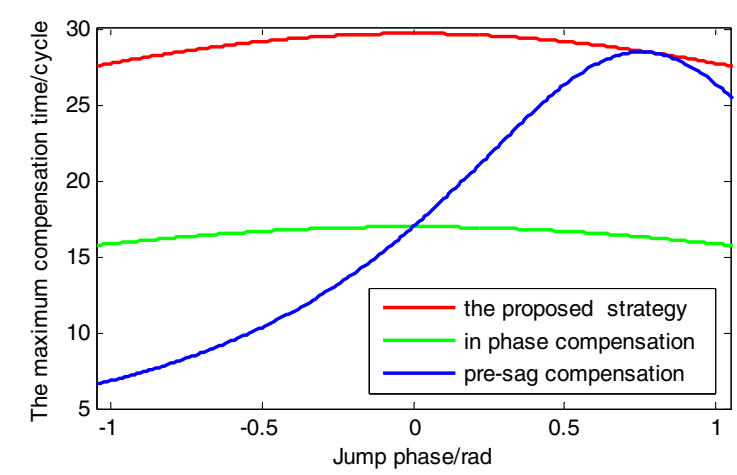

(b) The maximum compensation time and jump phase

Fig. 4 The maximum compensation time for different methods of the compensating voltage. Finally, the $U_{\text {pre-sag }}$ reaches to $U_{A-o p t}$.

\section{Results}

\section{Comparisons}

As mentioned above, the maximum compensation time plays an important role in reflecting the performance of DVR. It is directly connected to the amount of energy needed for the compensation and the needed capacitance. Based on the mentioned analytic equations, a comparative study is presented to compare the DVR maximum compensating time for different sags and jump phases by using the mentioned strategies (i.e. pre-sag strategy, in-phase strategy, and the proposed strategy.).

As shown in Fig. 1, a medium-voltage DVR system connected with a constant DC-link capacitor. In this system, the nominal grid voltage is $380 \mathrm{~V}$, the apparent load power is $9 \mathrm{kVA}$, the turn ratio is 1 , the maximum modulation index is set as 1 , the power factor is kept at 0.7 (lag), the phase jump is $45^{\circ}$ (positive) with a $50 \%$ given sag depth, and the line frequency is $50 \mathrm{~Hz}$ while the cycle is $20 \mathrm{~ms}$. Then the obtained capacitance of dc link is 7mF. Using Eq. (1), (12), (7), (13), (9), (11) and (18), As shown in Fig. 4, the maximum compensation time for the proposed strategy, in-phase compensation and presag compensation in different situation are compared. Figure 4(a) shows the maximum compensation time of different strategies with the change of sag depth. When the sag depth is given, Fig. 4(b) shows the maximum time with respect to the different jump angles by using different compensation strategies.

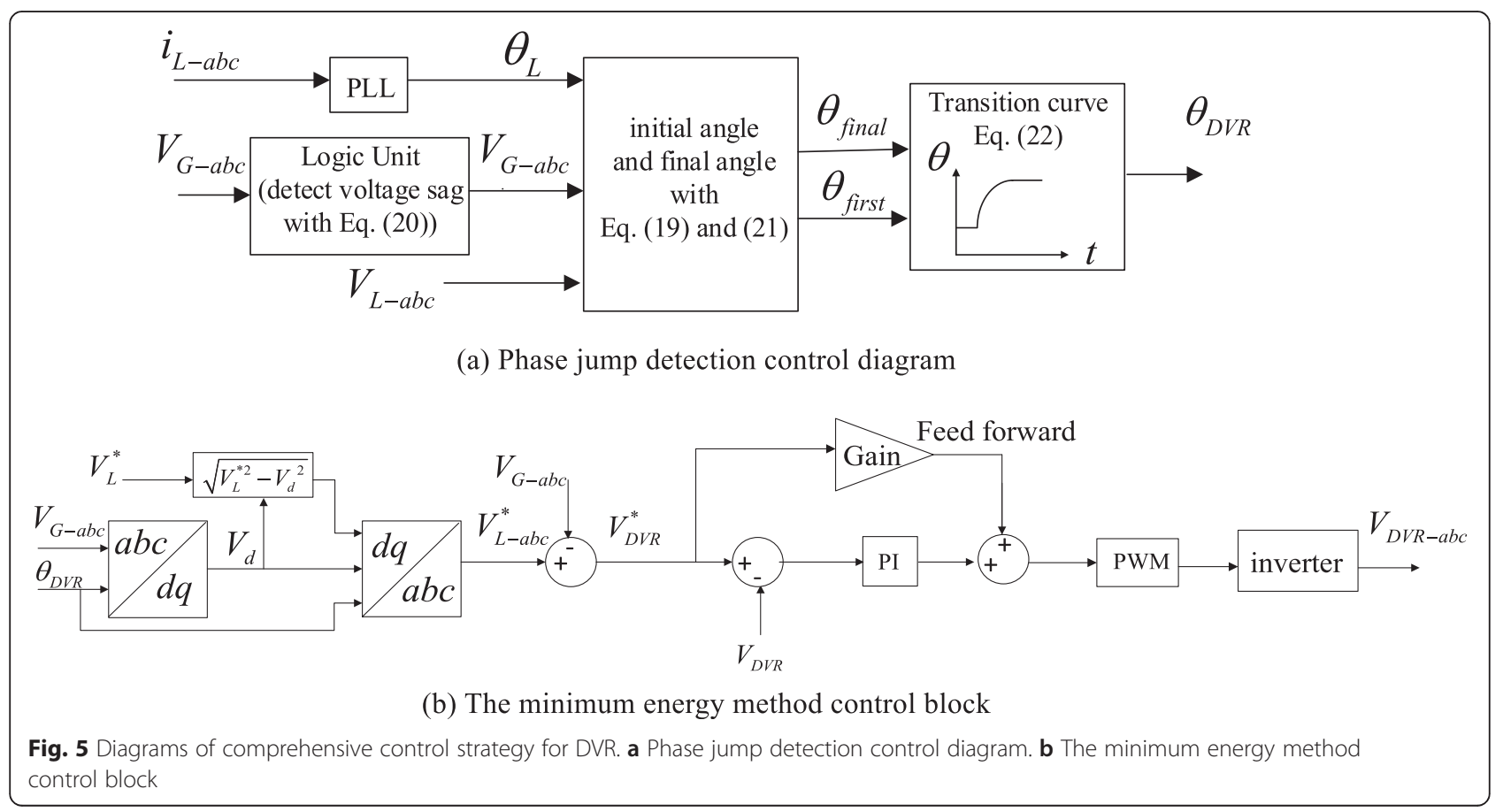




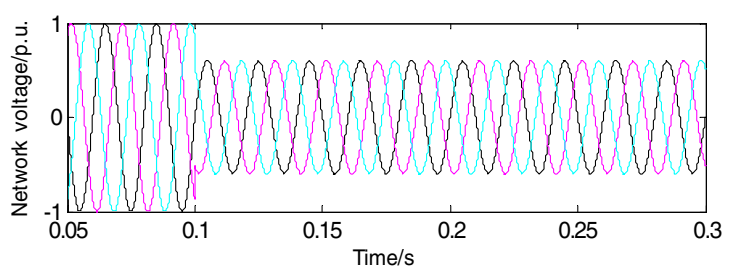

(a)

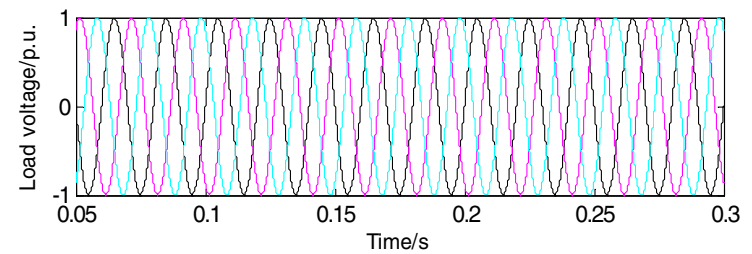

(b)

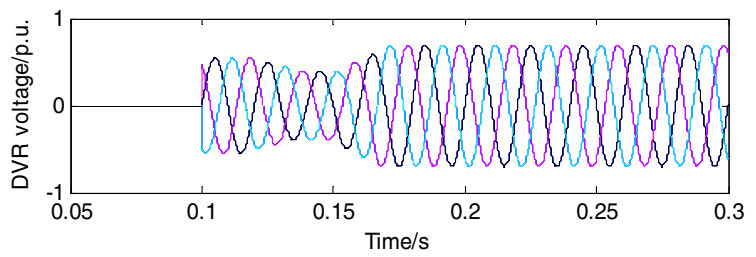

(c)

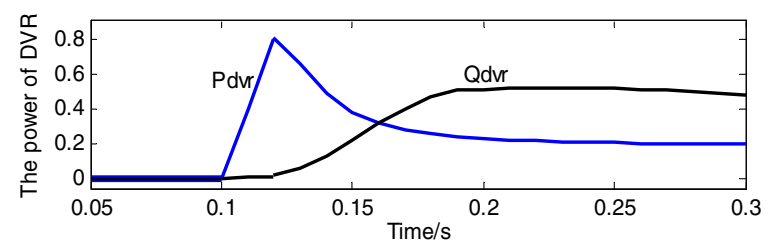

(d)

Fig. 6 Simulation results for $40 \%$ sag depth

The sag depth is varied from $10 \%$ to $80 \%$ of the rated voltage. As shown in Fig. 4(a), the proposed method has the longest supporting time among three strategies at the same sag depth. Taking $50 \%$ sag depth for example, the compensation time of the in-phase compensation is longer than that of the pre-sag compensation. The proposed method further increases the compensation time to $20 \mathrm{cy}$ cles based on in-phase compensation. When the sag depth is lower than $30 \%$, the proposed strategy works in the self-supporting mode. The minimize utilization of dc active power makes the slowest discharge of capacitor rate among three compensation methods, so the compensation time can be infinitely prolonged. Figure 4 (b) depicts that the phase jump is varied from $-60^{\circ}$ to $60^{\circ}$ for a $50 \%$ sag depth when other conditions don't change. The compensating time of in-phase compensation and the proposed compensation have no obvious fluctuation with the change of phase jump, while pre-sag compensation is opposite. The proposed strategy has the longest compensation time among three strategies.

\section{Simulation study}

\section{Block diagram}

The simulation complete setup is depicted in Fig. 1. The block diagrams of the comprehensive compensation strategy for DVR are shown in Fig. 5 in detail. Take a $380 \mathrm{~V}$ radial micro grid as an example, the sensitive loads is set as $R-L$ loads. Three phase symmetrical voltage drop with $40 \%$ sag depth happens in the grid. Among it, the phase jump is considered as $25^{\circ}$. The frequency is $50 \mathrm{~Hz}$, so the cycle is $20 \mathrm{~ms}$. The rest parameters of DVR are the same as that mentioned in section 4.

According to Eq. (20), the voltage sag in grid is monitored by logical unit. Control system includes two sub-modules, one is the calculation of the injected initial angle and final angle of DVR, and another is the transition to the minimum energy compensation. The line current, which is acted as a reference, can be gotten from PLL to realize the decoupling control of active and reactive power. For the phase angle detection module, PLL is used to calculate the DVR initial angle of pre-sag compensation and the final angle of the minimum energy compensation. After the transition, the reference voltage is given by the minimum energy module, i.e. $U_{L-a b c}^{*}=U_{o p t}$. The reference voltage $U_{D V R}^{*}$ is generated by DVR. $U_{D V R}^{*}$ is compared with the actual voltage $U_{D V R}$ in the stationary reference frame. The difference of $U_{D V R}$ and $U_{D V R}^{*}$ is tracked by the PI controller accurately. A feed forward control with gain is added to compensate the loss of DVR system.

\section{Simulation result}

The $\Delta t_{\text {tran }}$ in transition is taken as $40 \mathrm{~ms}$. From these, it can be obtained that the DVR initial injected angle is $16^{\circ}$ while the final angle is $81.5^{\circ}$. The simulation results are given in Fig. 6. Figure 6(a) shows the voltage sag happens at $0.1 \mathrm{~s}$. Figure 6(b) shows the amplitude and phase of load voltage return to normal after the recovery of DVR. Figure 6(c) shows the injected voltage of DVR during the sag. Figure 6(d) shows the power change of DVR during compensation.

As shown in Fig. 6, the sag depth was $40 \%$, and the start time of sag was $0.1 \mathrm{~s}$. The DVR injected voltage and power were shown in the last two graphs of Fig. 6. In the power graph, it was noticed that DVR injected a large number of active power to compensate the phase jump in first cycle of initial stage. After two cycles, presag compensation was gradually shifted to the minimum energy compensation mode. Accordingly, the injected voltage made a smooth transition, which can be seen from the third graph. In the last process, the injected active power was gradually reduced and became steady. After the recovery by DVR, the amplitude and phase of load voltage returned to normal.

\section{Conclusion}

To manage the power quality in a micro grid, an optimized compensation strategy of DVR has been proposed based 
on three basic strategies in the paper. The proposed strategy protects sensitive loads against the grid voltage sags accompanied with the phase jump. At the beginning of voltage sag, pre-sag compensation is adopted to detect and compensate phase jump. The full compensation of voltage amplitude and phase is achieved. After two cycles, pre-sag compensation is shifted to the minimum energy compensation to reduce the consumption of DVR active power. To mitigate the influence of phase jump on sensitive loads, the voltage phase is converted smoothly according to a quadratic transition curve. Although the presented method has good compensation effects to three-phase symmetrical voltage sags with different characteristics, a more accurate mathematical model needs to be established for unbalanced three-phase voltage drop. Furthermore, the compensation time of DVR under pre-sag compensation can be significantly increased when applying the proposed optimized strategy. In practical application, the proposed method can meet the efficient and accurate requirements of DVR.

\section{Acknowledgment}

This work is supported by National Nature Science Foundation under Grant 51477070, and the Priority Academic Program Development of Jiangsu Higher Education Institutions (PAPD).

\section{Authors' contributions}

ZML proposed the optimized compensation strategy of DVR for voltage sag, carried out a quadratic transition which involves the injected voltage phases of pre-sag strategy and minimum energy strategy, finished the related calculation of voltage amplitude and phase. WWL carried out the DVR compensating strategy studies, derived the maximum compensation time of DVR by control requirements, participated in the comparison of the maximum compensation time for different methods and drafted the manuscript. THP established the model and carried out the simulation study of the proposed compensation of DVR, gave the voltage and power change during the compensation, made the summary. All authors read and approved the final manuscript.

\section{About the Authors}

W.W. Li, received her B.S degree from Jiangsu University in 2013. Now she has been a postgraduate student in School of Electrical and Information Engineering, Jiangsu University, Zhenjiang, China. Her current research interests include micro-grid, power electronic devices.

Z. M. Li, received his B.S. degree from zhenjiang agricultural machinery institute and received his M.S degree from Xi'an Jiao Tong University in 1987. Now he has been a professor in School of Electrical and Information Engineering, Jiangsu University, Zhenjiang, China. His current research interests include industrial computer network, process control, power system remote monitoring etc. He published over 100 papers, more than 20 articles were included by $\mathrm{SCl}$ and $\mathrm{El}$. He obtained second prize for technological progress of the original ministry of machine and the second prize of scientific and technological progress of Jiangsu province, and outstanding teaching achievement prizes of Jiangsu province.

T.H. Pan, received his B.S. degree from Anhui Agriculture University and M.S. degree from Gansu University of Technology in 1997 and 2000 respectively. And he received his Ph.D. degree in control theory and control engineering from Shanghai Jiao Tong University in 2007. Now he has been a professor in School of Electrical \& Information Engineering, Jiangsu University, Zhenjiang, China. His current research interests include multiple model approach and its application, machine learning, virtual metrology, predictive control and Run-to-Run control theory and practice, etc.

\section{Competing interests}

The authors declare that they have no competing interests.
Received: 12 May 2016 Accepted: 16 May 2016

Published online: 02 July 2016

\section{References}

1. Wang, C., \& Li, P. (2010). Development and challenges of distributed generation, the micro-grid and smart distribution system [J]. Automation of Electric Power Systems, 34(2), 10-14.

2. Wang, C., \& Wang, S. (2008). Study on some key problems related to distributed generation systems [J]. Automation of Electric Power Systems, 32(20), 1-4.

3. Wang, C., Wu, Z., \& Li, P. (2014). Research on key technologies of microgrid [J]. Transactions of China Electrotechnical Society, 29(2), 1-4.

4. Kaniewski, J., Fedyczak, Z., \& Benysek, G. (2014). AC voltage sag/swell compensator based on three-phase hybrid transformer with buck-boost matrix-reactance chopper. IEEE Transactions on Industrial Electronics, 61(8), 3835-3846.

5. Castilla, M., Miret, J., Camacho, A., Matas, J., \& de Vicuna, L. (2014). Voltage support control strategies for static synchronous compensators under unbalanced voltage sags. IEEE Transactions on Industrial Electronics, 61(2), 808-820.

6. Kumar, C., \& Mishra, M. (2014). A multifunctional DSTATCOM operating under stiff source. IEEE Transactions on Industrial Electronics, 61(7), 3131-3136.

7. Wang, T., Xue, Y., \& Choi, S. S. (2007). Review of dynamic voltage restorer [J]. Automation of Electric Power Systems, 31(9), 101-107.

8. Liu, Y.-y., Xiao, X.-n., \& XU, Y.-h. (2010). Characteristics analysis on energy steady compensation for dynamic voltage restorer [J]. Proceedings of the CSEE, 30(13), 69-74.

9. Sadigh, A. K., \& Smedley, K. M. (2012). Review of voltage compensation methods in dynamic voltage restorer (DVR). In Proc. IEEE Power Energy Soc. Gen. Meet (pp. 1-8)

10. Feng, X.-m., \& Yang, R.-g. (2004). Analysis of voltage compensation strategies for dynamic voltage restorer (DVR) [J]. Automation of Electric Power Systems, 28(6), 68-72.

11. Wang, J., Xu, A.-q., Weng, G.-q., et al. (2010). A survey on control strategy of DVR [J]. Power System Protection and Control, 38(1), 145-151.

12. Al-Hadidi, H. K., Gole, A. M., \& Jacobson, D. A. (2008). A novel configuration for a cascade inverter based dynamic voltage restorer with reduced energy storage requirements. IEEE Transactions on Power Delivery, 23(2), 881-888.

13. Sun, Z., Guo, C., Xiao, X., et al. (2010). Analysis method of DVR compensation strategy based on load voltage and minimum energy control[J]. Proceedings of the CSEE, 30(31), 43-49.

14. Xiao, X.- n., Xu, Y.- h., \& Liu, L.- g. (2002). Research on mitigation methods of voltage sag with phase - angle jump [J]. Proceedings of the CSEE, 22(1), 64-69.

15. Meyer, C., Doncker, R. W., Li, Y. W., \& Blaabjerg, F. (2008). Optimized control strategy for a medium-voltage DVR-Theoretical investigations and experimental results. IEEE Transactions on Power Electronics, 23(6), 2746-2754.

16. Ke, C.-b., \& Li, Y.-I. (2012). Study on voltage sags compensation strategy for dynamic voltage restorer [J]. Power System Protection and Control, 40(17), 94-99.

\section{Submit your manuscript to a SpringerOpen ${ }^{\odot}$ journal and benefit from:}

- Convenient online submission

- Rigorous peer review

- Immediate publication on acceptance

- Open access: articles freely available online

- High visibility within the field

- Retaining the copyright to your article

Submit your next manuscript at springeropen.com 